\title{
Perfil epidemiológico e preditores de fatores de risco para a COVID-19 na região sul do Brasil
}

\author{
Epidemiological profile and risk factors predictors of COVID-19 in southern Brazil \\ Perfil epidemiológico y predictores de factores de riesgo para COVID-19 en el sur de Brasil
}

Recebido: 22/02/2021 | Revisado: 01/03/2021 | Aceito: 03/03/2021 | Publicado: 11/03/2021

Sarah Gisele Martins Klokner

ORCID: https://orcid.org/0000-0002-4074-873X

Universidade Federal de Santa Catarina, Brasil

E-mail: sarahgisele@outlook.com

Ramsés Antunes da Luz

ORCID: https://orcid.org/0000-0003-4046-9674

Universidade do Oeste de Santa Catarina, Brasil

E-mail: ramsesantunes@gmail.com

Pedro Henrique de Moura Araujo

ORCID: https://orcid.org/0000-0003-3657-8958

Instituto Nacional de Estudos e Pesquisas Educacionais Anísio Teixeira, Brasil

E-mail:phma05@gmail.com

Janete Knapik

ORCID: https://orcid.org/0000-0002-3449-1376

Universidade Federal de Santa Catarina, Brasil

E-mail: janete.knapik@up.edu.br

Synara Sepúlveda Sales

ORCID: https://orcid.org/0000-0002-7792-4952

Universidade Tuiuti do Paraná, Brasil

E-mail: synarasepulveda@hotmail.com

Grasiela Torrico

ORCID: https://orcid.org/0000-0002-1200-9876

Universidade Federal de Santa Catarina, Brasil

E-mail: grasit@hotmail.com

Fernanda Pereira Labiak

ORCID: https://orcid.org/0000-0002-8272-711X

Universidade Federal de Santa Catarina, Brasil

E-mail: fernandalabiak@gmail.com

Maria Julia Pegoraro Gai

ORCID: https://orcid.org/0000-0002-8481-1112

Universidade Federal de Santa Catarina, Brasil

E-mail: mariajuliagai@ hotmail.com

Clarissa Venturieri

ORCID: https://orcid.org/0000-0001-5322-679X

Universidade Federal de Santa Catarina, Brasil

E-mail: c.venturieri@gmail.com

Rafael Frasson

ORCID: https://orcid.org/0000-0002-6658-979X

Universidade do Sul de Santa Catarina, Brasil

E-mail: psicologorafaelfrasson@gmail.com

Adelino Domingos Onofre

ORCID: https://orcid.org/0000-0002-5262-7572

Universidade Federal de Santa Catarina, Brasil

E-mail: adelinoonofre@mail.com

Roberto Moraes Cruz

ORCID: https://orcid.org/0000-0003-4671-3498

Universidade Federal de Santa Catarina, Brasil

E-mail: robertocruzdr@gmail.com

\begin{abstract}
Resumo
Detectar fatores associados ao risco ou à proteção para a COVID-19 é imprescindível para se determinar possíveis políticas públicas de prevenção relacionadas às condições epidêmicas. O objetivo deste estudo é analisar o perfil epidemiológico, bem como analisar as variáveis preditoras de risco e proteção para a COVID-19 na região sul do Brasil. Foi realizado um estudo epidemiológico, com base em uma série temporal de 34 semanas e a partir da base de dados sobre a COVID-19 provenientes das Secretarias de Estado da Saúde do Rio Grande do Sul, Santa Catarina e
\end{abstract}


Paraná. Foram utilizados cálculos de prevalência, incidência, tabelas de frequência, teste qui-quadrado, razão de chance (odds ratio) e regressão binária por meio do programa estatístico SPSS, v.22. Os resultados deste estudo indicaram que as variáveis estado, sexo, faixa etária e comorbidade predizem a condição de pessoas contaminadas de se recuperarem, falecerem, manifestarem sintomas ou serem internadas em virtude da COVID-19.

Palavras-chave: COVID-19; Pandemia; Perfil epidemiológico; Razão de chances; Risco.

\begin{abstract}
Detecting factors associated with risk or protection for COVID-19 is essential to determine possible public prevention policies related to epidemic conditions. The objective of this study is to analyze the epidemiological profile, as well as to analyze the predictive variables of risk and protection for COVID-19, in the southern region of Brazil. An epidemiological study was carried out, based on a 34-week time series and based on the database on COVID-19 from the -19 from the State Secretariats of Health of Rio Grande do Sul, Santa Catarina and Paraná, Brazil. Calculations of prevalence, incidence, frequency tables, chi-square test, odds ratio and binary regression were used using the SPSS statistical program, v.22. The results of this study indicated that the variables state, sex, age and comorbidity predict the condition of infected people to recover, die, manifest symptoms or be hospitalized due to COVID-19.
\end{abstract}

Keywords: COVID-19; Pandemic; Epidemiological profile; Odds Ratio; Risk.

\title{
Resumen
}

La detección de factores asociados a riesgo o protección por COVID-19 es fundamental para determinar posibles políticas públicas de prevención relacionadas con condiciones epidémicas. El objetivo de este estudio es analizar el perfil epidemiológico, así como analizar las variables predictivas de riesgo y protección para COVID-19 en la región sur de Brasil. Se realizó un estudio epidemiológico, basado en una serie de tiempo de 34 semanas y con base en la base de datos sobre COVID-19 de las Secretarías de Salud de los Estados de Rio Grande do Sul, Santa Catarina y Paraná. Se utilizaron los cálculos de prevalencia, incidencia, tablas de frecuencia, prueba de chi-cuadrado, razón de probabilidades y regresión binaria mediante el programa estadístico SPSS, v.22. Los resultados de este estudio indicaron que las variables estado, sexo, edad y comorbilidad predicen la condición de las personas infectadas para recuperarse, morir, manifestar síntomas o ser hospitalizadas por COVID-19.

Palabras clave: COVID-19; Pandemia; Perfil epidemiológico; Razón de probabilidades; Riesgo.

\section{Introdução}

A pandemia provocada pela COVID-19 estimulou a adoção de medidas de isolamento e distanciamento social, em diferentes países, como forma de conter o avanço da contaminação e os efeitos severos da doença (WHO, 2020). Apesar disso, na maior parte da região sul do Brasil, o intenso e veloz alastramento da COVID-19 causou uma sobrecarga nos hospitais e nas Unidades de Terapia Intensiva (UTIs). Inferências baseadas em epidemiologia descritiva sobre o comportamento da pandemia nessa região - tais como a prevalência e incidência - tornam-se cruciais para auxiliar no monitoramento e evolução da doença, assim como fornecer indicadores em saúde pública.

Ações voltadas à prevenção, ao tratamento dos infectados e ao controle dos efeitos da COVID-19 dependem de um conhecimento cada vez mais específico de aspectos clínicos e epidemiológicos da doença. Modelos matemáticos e análises estatísticas robustas sobre o desempenho dos dados produzidos por pesquisadores e pelas equipes de saúde na pandemia contribuem para a tomada de decisão sobre o planejamento e controle da doença e suas consequências (Giordano et al., 2020).

O estudo de preditores de desfechos da COVID-19 contribui o conhecimento sobre a influência de fatores de risco e proteção na população em risco de contaminação, colaborando na gestão das políticas de saúde pública (Verity et al., 2020). À medida que o conhecimento sobre o curso clínico epidemiológico da COVID-19 avança, o entendimento dos fatores de risco e proteção para doenças graves têm sido aprimorados, assim como as estimativas das populações de maior risco (Centers for Disease Control and Prevention, 2020).

Em epidemiologia é possível predizer a ocorrência de um agravo à saúde - em um período determinado - por meio da mensuração da magnitude da associação entre o agravo e uma condição (ou fator) de risco supostamente relacionada a ele, o que demonstraria a chance de um evento ocorrer ou ser predito. Algumas condições podem ser consideradas como preditoras, visto que a sua presença pode influenciar diretamente variáveis relacionadas a uma doença, ampliando-as ou mitigando-as. São denominados fatores de risco aqueles relacionados às características sociodemográficas, fatores pessoais ou de exposição 
ambiental, associadas ao aumento da probabilidade de ocorrência de alguma doença, enquanto os fatores de proteção são aqueles que contribuem para a sua atenuação. Uma vez que os fatores de risco podem ser identificados, as medidas que os atenuam podem diminuir a ocorrência de doenças (Bonita et al., 2010).

Estudos recentes destacam alguns fatores preditores de risco à infecção por COVID-19 na população. Entre esses fatores preditores de risco conhecidos, associados a resultados clínicos graves desta doença, estão incluídos: idade avançada, doenças pulmonares crônicas, estado imunocomprometido e outras comorbidades mais prevalentes, como hipertensão, diabetes ou doença cardiovascular (Emami et al., 2020; Zheng et al., 2020).

Outros fatores de risco potencialmente preditores são o câncer e as doenças respiratórias, os quais podem desempenhar um papel singular no agravamento do impacto da pandemia, sendo responsáveis pela maior parte das mortes em todo o mundo (Singh et al., 2020). O tabagismo também pode ser incluído como um preditor de fator de risco, pois os pacientes fumantes do sexo masculino com mais de sessenta e cinco anos possuem uma chance maior da COVID-19 evoluir para uma condição crítica ou mortal (Zheng et al., 2020).

As mulheres são menos suscetíveis à infecção viral do que os homens, possivelmente por causa da proteção do cromossomo X e hormônios sexuais, que desempenham um papel essencial na imunidade inata e adaptativa (Cutolo, 2006). Entre os fatores preditores sociodemográficos, a disseminação da doença mostrou-se mais intensa em núcleos urbanos próximos a polos econômicos e em cidades adjacentes em função do fluxo intenso de pessoas e cargas (Guimarães et al., 2020).

\section{Metodologia}

Foi realizado um estudo de natureza quantitativa (Pereira, Shitsuka, Parreira, \& Shitsuka, 2018) e delineamento descritivo e inferencial voltados à análise do perfil epidemiológico e dos fatores preditores de risco e proteção à COVID-19 nos estados do Rio Grande do Sul, de Santa Catarina e do Paraná. Os bancos de dados utilizados são de domínio público, disponibilizados em plataformas provenientes do Governo do Estado do Rio Grande do Sul (2020), de Santa Catarina (2020) e do Paraná (2020). Foi considerada uma série temporal de 34 semanas, correspondente ao período de 26 fevereiro de 2020 a 17 de outubro de 2020.

O Ministério da Saúde recebeu a primeira notificação de um caso confirmado de COVID-19 no Brasil no dia 26 de fevereiro de 2020 e, até o dia 17 de outubro de 2020 - data final da coleta de dados deste estudo realizado na região sul do Brasil - foram confirmados 5.224.362 casos, 153.675 óbitos e um coeficiente de incidência de 24.861 casos para cada 1 milhão de habitantes para COVID-19 no país (Ministério da Saúde, 2020b).

Dentre as variáveis que compõem os bancos de dados dos três estados, foram selecionadas: a) variáveis preditoras: estado (unidade da federação), faixa etária, sexo e comorbidade (presença de pelo menos uma destas doenças: asma, câncer, diabetes, diabetes mellitus, doença cardiovascular crônica, doença hematológica crônica, doença hepática crônica, doença neurológica crônica, doença pneumática crônica, doença renal crônica, doenças cardíacas crônicas, doenças renais crônicas em estágio avançado (graus 3, 4 ou 5), doenças respiratórias crônicas descompensadas, hipertensão, imunodeficiência, imunodepressão, imunossupressão, obesidade, outra pneumatopatia crônica, portador de doenças cromossômicas ou estado de fragilidade imunológica, Síndrome de Down); e b) variáveis desfechos: sintomas relacionados a COVID-19 (dor de cabeça, febre e tosse), internação (na instituição hospitalar e em UTI), recuperação e óbito. Para compatibilização dos dados, as variáveis foram categorizadas no nível nominal, com exceção da variável faixa etária, que foi transformada em ordinal e organizadas em quatro faixas etárias: 0 a 19 anos, 20 a 39 anos, 40 a 59 anos e 60 anos ou mais.

A análise de perfil epidemiológico foi viabilizada por meio da descrição e comparação entre as taxas de prevalência e incidência (Bonita et al., 2010; Pereira, 2013) de casos ativos acumulados nos três estados da região Sul. Os resultados sobre 
prevalência e incidência foram calculados para cada 100 mil habitantes. O número da população estimada por estado foi coletado com base nas informações do Instituto Brasileiro de Geografia e Estatística, sendo 11.422.973 habitantes no Rio Grande do Sul, 7.252.502 habitantes em Santa Catarina e 11.516.840 habitantes do Paraná (IBGE, 2020a).

$\mathrm{Na}$ análise dos preditores de fatores de risco e proteção observa-se que no banco de dados fornecido pela Secretaria da Saúde do Estado do Paraná algumas informações sobre as variáveis sintomas, internação e comorbidades estavam ausentes, o que inviabilizou parte das comparações desses aspectos com os demais estados da região Sul. No banco do estado do Paraná foram verificados 157 casos de datas de início de sintomas inexistentes ou com prováveis erros de digitação. Para sanar tais ausências foi considerada uma média de 14 dias entre a data de divulgação dos resultados e a data de início dos sintomas, permitindo assim uma comparação desta variável com a dos demais estados.

No banco de dados do estado do Rio Grande Sul foram desconsiderados do escopo da comorbidade as condições de gestação e puérperas. No banco de dados catarinense havia a distinção entre a ocorrência de internação em instituição hospitalar e em UTI, diferentemente dos demais estados. Dessa forma, para permitir a comparação entre as informações relativas às variáveis dos três estados optou-se por convertê-las em variáveis dummy (variáveis binárias 0 ou 1) para sinalizar se pertencem - ou não - a um determinado grupo. Exemplo: grupo de pessoas contaminadas que possuem ou não sintomas e, assim sucessivamente.

O banco de dados foi organizado em uma planilha do Microsoft Excel e foi tratado por meio do software estatístico Statistical Package for the Social Sciences (SPSS, versão 22). Os resultados foram obtidos a partir de cálculos de prevalência, incidência, teste qui-quadrado, razão de chance (odds ratio) e regressão binária. As razões de chance foram obtidas a partir de um intervalo de confiança de 95\% e com nível de significância menor que 0,05 .

\section{Resultados}

Os resultados foram organizados em duas partes. A primeira aborda informações epidemiológicas descritivas referentes às taxas de prevalência e incidência da COVID-19 nos três estados da região sul. Em sequência realiza-se o cálculo da razão de risco e proteção (odds ratio) demarcando os preditores epidemiológicos.

\subsection{Prevalência e incidência de casos ativos da COVID-19}

Taxas de prevalência e incidência contribuem para o diagnóstico do curso de uma pandemia, pois o cálculo das medidas de frequência de uma determinada doença depende de uma estimativa adequada do número de pessoas infectadas (ou ainda se infectando) em uma determinada população (Bonita et al., 2010). Os índices de prevalência e incidência de casos ativos acumulados nos três estados da região sul foram comparados em uma série temporal de 34 semanas, com o intuito de evidenciar diferenças ou similaridades na magnitude, distribuição e ocorrência da COVID-19 (Figura 1 e 2). 
Figura 1 - Taxas de prevalência casos ativos acumulados da COVID-19 no Rio Grande do Sul, Santa Catarina e Paraná.

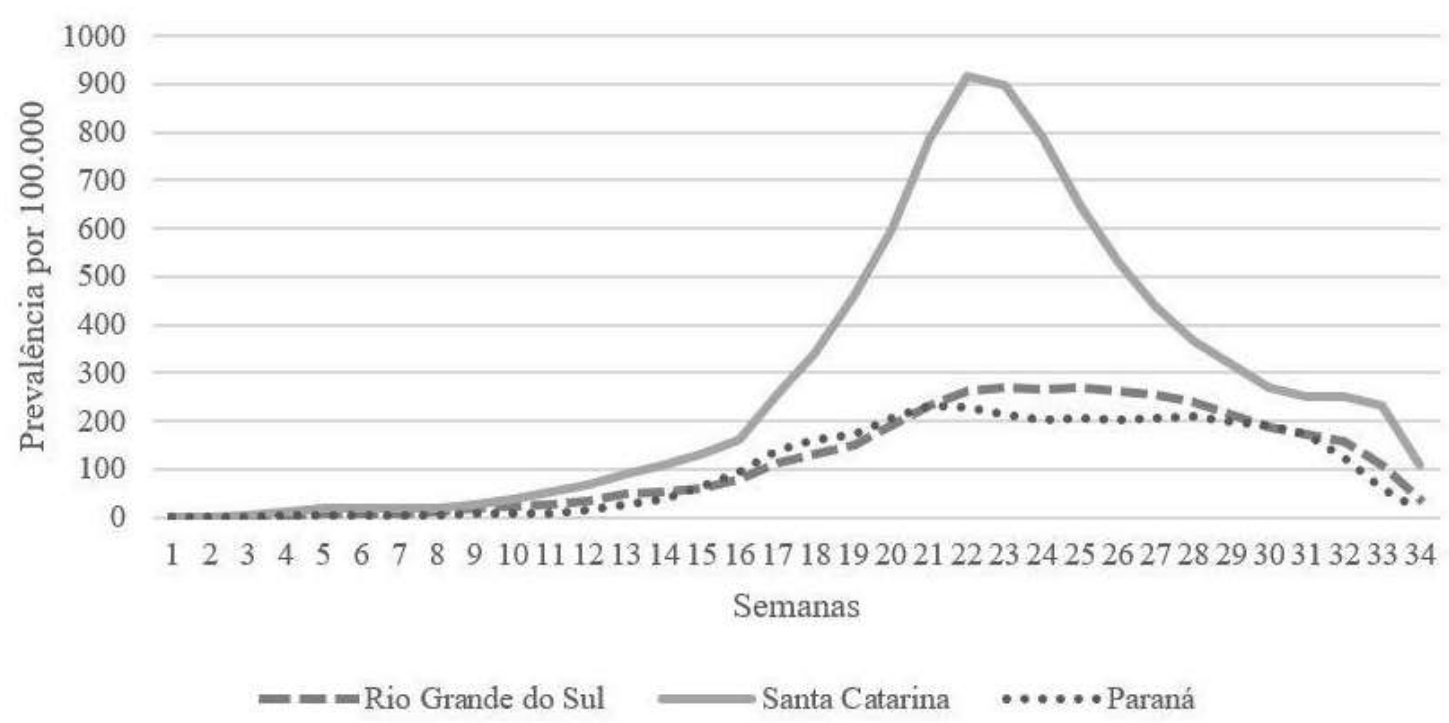

Fonte: Dados da pesquisa (2020).

A comparação entre as taxas de prevalência de casos ativos acumulados de COVID-19 no Rio Grande do Sul, Santa Catarina e Paraná evidencia uma tendência linear similar nos três estados até a décima semana. A partir deste período, inicia-se nas três áreas de ocorrência uma progressão ascendente indicando uma evolução do ritmo da pandemia com destaque para Santa Catarina. A partir da décima sexta semana percebe-se claramente que as taxas de prevalência de casos ativos da COVID19 em Santa Catarina cresceram acentuadamente em relação aos demais índices do Rio Grande do Sul e Paraná, atingindo seu ápice na vigésima segunda semana. Dessa data em diante, os indicadores dos três estados iniciaram uma desaceleração até a trigésima quarta semana. Contudo, tal decréscimo foi mais acentuado em Santa Catarina. Ao final da trigésima quarta semana tais índices passaram a se tornar similares (Figura 1). Essa diminuição das taxas de prevalência de casos ativos da COVID-19 nos estados do Rio Grande do Sul, Santa Catarina e Paraná a partir da vigésima segunda semana pode estar associada às medidas que foram tomadas para conter a pandemia.

Figura 2 - Taxas de incidência de casos ativos acumulados no Rio Grande do Sul, Santa Catarina e Paraná.

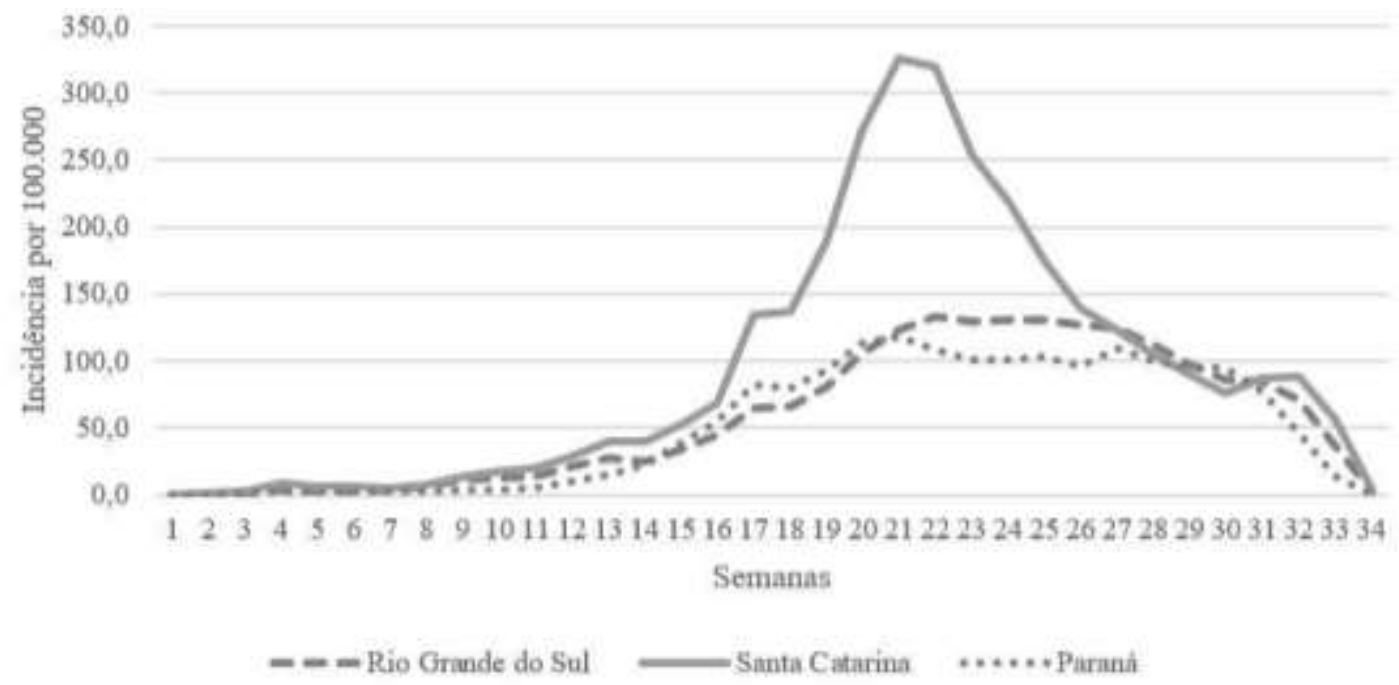

Fonte: Dados da pesquisa (2020). 
Ao comparar as taxas de incidência de casos ativos da COVID-19 entre os três estados da região sul do Brasil no período considerado, observa-se um comportamento semelhante ao que ocorreu com a prevalência. Há uma tendência conjunta do aumento de casos novos até a décima sexta semana. A partir desse período observa-se que as taxas de incidência de COVID-19 em Santa Catarina iniciam uma evolução progressiva acima dos níveis dos demais estados, até seu apogeu na vigésima segunda semana. A partir dessa data, os três estados sofrem uma redução progressiva até a trigésima quarta semana. Provavelmente houve, a partir da vigésima segunda semana, um maior controle e contenção da doença nos estados.

\subsection{Fatores de risco da COVID-19 na região sul do Brasil}

Fatores de risco ou proteção são condições associadas ao aumento ou diminuição da possibilidade de se desenvolver uma doença e abrangem qualquer situação que interfira na probabilidade de ocorrência deste agravo à saúde. Neste estudo foram calculados os coeficientes de risco ou proteção para três conjuntos de fatores: estado e sexo; estado e faixa etária; estado e comorbidade, comparados a quatro variáveis de desfecho: recuperações, óbito, sintomas - relacionados à COVID-19 - e internações. Os resultados foram obtidos a partir de um intervalo de confiança de 95\% e com nível de significância menor que 0,05 .

Tabela 1 - Prevalência e razão de chance, segundo o estado e sexo.

\begin{tabular}{|c|c|c|c|c|c|c|c|c|c|}
\hline \multirow{2}{*}{ Desfechos - } & \multicolumn{2}{|c|}{ Preditor } & \multirow[b]{2}{*}{$\mathbf{N}$} & \multicolumn{3}{|c|}{ Estatisticas } & \multicolumn{3}{|c|}{ IC $(95 \%)$} \\
\hline & Est. & Sexo & & Prev.(\%) & $x^{2}$ & $p$ & RC & Inf. & Sup. \\
\hline \multirow[t]{6}{*}{ Recuperadc } & RS & $\mathrm{F}$ & 100.913 & 94,5 & 110,06 & 0 & 1,011 & 1,009 & 1,014 \\
\hline & & M & 104.630 & 93,4 & & & & & \\
\hline & $\mathrm{SC}$ & $\mathrm{F}$ & 110.356 & 95,8 & 121,8 & 0 & 1,01 & 1,009 & 1,012 \\
\hline & & M & 102.583 & 94,8 & & & & & \\
\hline & PR & $\mathrm{F}$ & 100.381 & 98,1 & 305,64 & 0 & 1,013 & 1,011 & 1,014 \\
\hline & & M & 89.348 & 96,9 & & & & & \\
\hline \multirow[t]{6}{*}{ Óbito } & RS & $\mathrm{F}$ & 2.331 & 2,2 & 47,44 & 0 & 0,828 & 0,785 & 0,874 \\
\hline & & M & 2.951 & 2,6 & & & & & \\
\hline & $\mathrm{SC}$ & $\mathrm{F}$ & 1.165 & 1 & 170,11 & 0 & 0,616 & 0,573 & 0,663 \\
\hline & & M & 1.775 & 1,6 & & & & & \\
\hline & PR & F & 1.942 & 1,9 & 305,64 & 0 & 0,606 & 0,572 & 0,641 \\
\hline & & M & 2.890 & 3,1 & & & & & \\
\hline \multirow[t]{4}{*}{ Sintomas } & RS & $\mathrm{F}$ & 63.136 & 59,1 & 30,41 & 0 & 1,02 & 1,013 & 1,027 \\
\hline & & M & 64.911 & 57,9 & & & & & \\
\hline & SC & $\mathrm{F}$ & 72.053 & 62,6 & 96,27 & 0 & 1,033 & 1,027 & 1,04 \\
\hline & & M & 65.487 & 60,5 & & & & & \\
\hline \multirow[t]{4}{*}{ Internação } & RS & $\mathrm{F}$ & 8.168 & 7,6 & 84,61 & 0 & 0,876 & 0,852 & 0,901 \\
\hline & & M & 9.775 & 8,7 & & & & & \\
\hline & SC & $\mathrm{F}$ & 4.330 & 3,8 & 470,7 & 0 & 0,659 & 0,635 & 0,685 \\
\hline & & $\mathrm{M}$ & 6.170 & 5,7 & & & & & \\
\hline
\end{tabular}

* Obs.: $\chi 2$ = Qui-Quadrado de Pearson; $\mathrm{p}<0,05 ; \mathrm{RC}=$ Razão de Chances; $\mathrm{IC}=$ Intervalo de Confiança Fonte: Dados da pesquisa.

Verifica-se nos três estados pesquisados que infectados do sexo feminino detém maiores prevalências (RS: 94,5\%; SC: 95,8\%; PR: 98,1\%) do que as do sexo masculino (RS: 93,4\%; SC: 94,8\%; PR: 96,9\%) no que se refere a variável relativa à recuperação desta doença. Observa-se que as mulheres apresentam maiores chances de recuperação nas seguintes taxas: no 
Rio Grande do Sul a razão de chance é de 1,011 ( $\mathrm{p}=0,000$ IC=1,009-1,014), em Santa Catarina é de 1,010 ( $\mathrm{p}=0,000$ IC=1,0091,012) e no Paraná é de 1,013 (p=0,000 IC=1,011-1,014), índices similares entre os estados. Dessa forma, constata-se que sexo se constitui em uma variável preditora para a recuperação da COVID-19, evidenciando chances maiores para o sexo feminino.

Observa-se, nos três estados, que a prevalência de óbitos ocasionados pela COVID-19 é menor para o sexo feminino, assim como são menores as chances de óbito nas seguintes taxas: no Rio Grande do Sul a razão de chance é de 0,828 ( $\mathrm{p}=0,000$ IC=0,785-0,874), em Santa Catarina é de 0,573 ( $\mathrm{p}=0,000$ IC=0,573-0,663) e no Paraná é de 0,606 ( $\mathrm{p}=0,000$ IC=0,572-0,641).

Por outro lado, a prevalência de sintomas da COVID-19 é maior entre os contaminados do sexo feminino nos dois estados nos quais foi possível obter os dados (RS e SC). Observa-se que as mulheres apresentam maiores chances de manifestarem sintomas desta doença nas seguintes taxas: no Rio Grande do Sul a razão de chance é de 1,020 ( $\mathrm{p}=0,000$

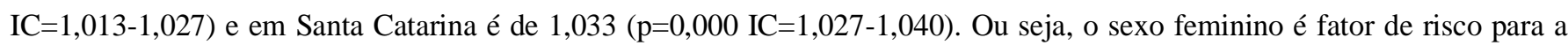
possibilidade de apresentar sintomas da COVID-19.

As prevalências de internação por COVID-19 são menores para o sexo feminino, assim como as mulheres apresentam menores chances de serem internadas nas seguintes taxas: no Rio Grande do Sul a razão de chance é de 0,876 ( $\mathrm{p}=0,000$ IC=0,852-0,901) e em Santa Catarina é de 0,659 (p=0,000 IC=0,635-0,685), indicando maior proteção para Santa Catarina. Assim, se constata que pertencer ao sexo feminino é considerado fator de proteção para internação por COVID-19. 
Tabela 2 - Prevalência e razão de chance, segundo o estado e a faixa etária.

\begin{tabular}{|c|c|c|c|c|c|c|c|c|c|}
\hline \multirow{2}{*}{ Desfecho } & \multicolumn{2}{|r|}{ Preditor } & \multirow[b]{2}{*}{$\mathbf{N}$} & \multicolumn{3}{|c|}{ Estatistica } & \multirow[b]{2}{*}{ RC } & \multicolumn{2}{|c|}{ IC $(95 \%)$} \\
\hline & Est. & Faixa Etári & & Prev. $(\%)$ & $x^{2}$ & $p$ & & Inf. & Sup. \\
\hline \multirow{12}{*}{ Recuperadc } & \multirow{4}{*}{ RS } & 0 a 19 anos & 19164 & 96,3 & \multirow{4}{*}{6382,4} & 0 & 1 & . & . \\
\hline & & 20 a 39 ano: & 85794 & 96,2 & & 0,467 & 0,97 & 0,895 & 1,052 \\
\hline & & 40 a 59 ano: & 70332 & 95,4 & & 0 & 0,803 & 0,741 & 0,871 \\
\hline & & $\geq 60$ anos & 30253 & 84,1 & & 0 & 0,204 & 0,189 & 0,221 \\
\hline & \multirow{4}{*}{$\csc$} & 0 a 19 anos & 19001 & 96,8 & \multirow{4}{*}{3757,8} & 0 & 1 & - & $\cdot$ \\
\hline & & 20 a 39 ano: & 98668 & 96,5 & & 0,093 & 0,929 & 0,853 & 1,012 \\
\hline & & 40 a 59 ano: & 71706 & 96,2 & & 0 & 0,847 & 0,776 & 0,925 \\
\hline & & $\geq 60$ anos & 23559 & 87,5 & & 0 & 0,234 & 0,214 & 0,255 \\
\hline & \multirow{4}{*}{ PR } & 0 a 19 anos & 19211 & 99,9 & \multirow{4}{*}{6870,1} & 0 & 1 & - & - \\
\hline & & 20 a 39 ano: & 84325 & 99,8 & & 0,001 & 0,448 & 0,283 & 0,71 \\
\hline & & 40 a 59 ano: & 63222 & 98.5 & & 0 & 0,067 & 0,043 & 0,104 \\
\hline & & $\geq 60$ anos & 22971 & 86,3 & & 0 & 0,007 & 0,004 & 0,01 \\
\hline \multirow{12}{*}{ Obitos } & \multirow{4}{*}{$\mathrm{RS}$} & 0 a 19 anos & 15 & 0,1 & \multirow{4}{*}{6706,6} & 0 & 1 & - & . \\
\hline & & 20 a 39 ano: & 163 & 0,2 & & 0,001 & 2,427 & 1,43 & 4,12 \\
\hline & & 40 a 59 ano: & 865 & 1,2 & & 0 & 15,744 & 9,448 & 26,235 \\
\hline & & $\geq 60$ anos & 4239 & 11,8 & & 0 & 177,05 & 106,606 & 294,03 \\
\hline & \multirow{5}{*}{ SC } & 0 a 19 anos & 10 & 0,1 & \multirow{4}{*}{4467} & 0 & 1 & - & - \\
\hline & & 20 a 39 ano: & 93 & 0,1 & & 0,081 & 1,787 & 0,931 & 3,432 \\
\hline & & 40 a 59 ano: & 554 & 0,7 & & 0 & 14,697 & 7,862 & 27,473 \\
\hline & & $\geq 60$ anos & 2283 & 8,5 & & 0 & 181,81 & 97,665 & 338,46 \\
\hline & & 0 a 19 anos & 20 & 0,1 & \multirow{4}{*}{6870,1} & 0 & 1 & - & - \\
\hline & \multirow{3}{*}{ PR } & 20 a 39 ano: & 196 & 0,2 & & 0,001 & 2,333 & 1,409 & 3,538 \\
\hline & & 40 a 59 ano: & 982 & 1.5 & & 0 & 1492 & 9.58 & 23,236 \\
\hline & & $\geq 60$ anos & 3634 & 13,7 & & 0 & 151,96 & 97,878 & 235,92 \\
\hline \multirow{8}{*}{ Sintomas } & \multirow{5}{*}{ RS } & 0 a 19 anos & 9129 & 459 & \multirow{4}{*}{1825,4} & 0 & 1 & - & $\cdot$ \\
\hline & & 20 a 39 ano: & 51242 & 57,4 & & 0 & 1,593 & 1,545 & 1,643 \\
\hline & & 40 a 59 ano: & 44965 & 61 & & 0 & 1,846 & 1,788 & 1,905 \\
\hline & & $\geq 60$ anos & 22711 & 63,1 & & 0 & 2,019 & 1,949 & 2,091 \\
\hline & & 0 a 19 anos & 10231 & 52,1 & & 0 & 1 & & \\
\hline & $\mathrm{SC}$ & 20 a 39 ano: & 63390 & 62 & 880 & 0 & 1,501 & 1,455 & 1,548 \\
\hline & No & 40 a 59 ano: & 46430 & 62,3 & , & 0 & 1,519 & 1,471 & 1,568 \\
\hline & & $\geq 60$ anos & 17489 & 649 & & 0 & 1,703 & 1,641 & 1,769 \\
\hline & & 0 a 19 anos & 245 & 1,2 & & 0 & 1 & & \\
\hline & PS & 20 a 39 ano: & 2144 & 2,4 & 161822 & 0 & 1,976 & 1,73 & 2,257 \\
\hline & No & 40 a 59 ano: & 5688 & 7,7 & 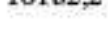 & 0 & 6,709 & 5,898 & 7,632 \\
\hline Int & & $\geq 60$ anos & 9866 & 27,4 & & 0 & 30,31 & 26,664 & 34,45 \\
\hline 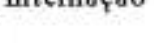 & & 0 a 19 anos & 150 & 0,8 & & 0 & 1 & - & - \\
\hline & 8 & 20 a 39 ano: & 1438 & 1,4 & 10 & 0 & 1,85 & 1,566 & 2,195 \\
\hline & se & 40 a 59 ano: & 3649 & 4,9 & 10912,0 & 0 & 6,687 & 5,675 & 7,88 \\
\hline & & $\geq 60$ anos & 5263 & 19,5 & & 0 & 31,559 & 26,801 & 37,163 \\
\hline
\end{tabular}

Obs.: $\chi 2$ = Qui-Quadrado de Pearson; $\mathrm{p}<0,05 ; \mathrm{RC}=$ Razão de Chances; IC= Intervalo de Confiança. Fonte: Dados da pesquisa.

Observa-se, nos três estados, que a prevalência de recuperação da COVID-19 é menor na faixa etária de 60 anos, e também com menores chances de recuperação nas seguintes taxas: no Rio Grande do Sul a razão de chance é de 0,20 ( $\mathrm{p}=0,000$ IC $=0,19-0,22)$, em Santa Catarina é de 0,23 ( $\mathrm{p}=0,000 \mathrm{IC}=0,21-0,25)$ e no Paraná é de 0,07 ( $\mathrm{p}=0,000$ IC=0,004-0,010). A 
menor probabilidade de recuperação é no Paraná.

No caso de óbitos provocados pela COVID-19, as prevalências são maiores na faixa etária de 60 anos ou mais, assim como se constitui em um fator de risco importante. No Rio Grande do Sul a razão de chance para óbito por COVID-19 é de 177,046 ( $\mathrm{p}=0,000$ IC=106,606-294,027), em Santa Catarina é de 181,814 ( $\mathrm{p=0,000} \mathrm{IC=97,665-338,463)} \mathrm{e} \mathrm{no} \mathrm{Paraná} \mathrm{é} \mathrm{de}$ 151,958 ( $\mathrm{p}=0,000$ IC=97,878-235,920), acentuando-se, portanto, em Santa Catarina. De igual forma, observa-se que a faixa etária de 60 anos ou mais apresenta maiores taxas de prevalência da variável sintomas da COVID-19, nos dois estados em que foi possível investigar: no Rio Grande do Sul a razão de chance é de 2,019 ( $\mathrm{p}=0,000$ IC=1,949-2,091) e em Santa Catarina é de 1,703 ( $\mathrm{p}=0,000 \mathrm{IC}=1,641-1,769$ ), constituindo-se em um importante fator de risco para a COVID-19, acentuadamente na população do Rio Grande do Sul.

Nos dois estados pesquisados as prevalências de internação da faixa etária de 60 anos ou mais (RS: 27,4\%; SC: 19,5\%) são maiores que as demais faixas. Esta faixa apresentou maiores chances de internação nas seguintes taxas: no Rio Grande do Sul a razão de chance é de 30,31 (p=0,000 IC=26,664-34,450) e em Santa Catarina é de 31,559 (p=0,000 IC=26,801-37,163), indicando maior risco para Santa Catarina. Assim, se constata que a faixa etária é uma variável preditora para internação pela COVID-19, sinalizando uma chance maior (fator de risco) para as pessoas com 60 anos ou mais nos estados em questão.

Tabela 3 - Prevalência e razão de chance, segundo o estado e comorbidade.

\begin{tabular}{|c|c|c|c|c|c|c|c|c|c|}
\hline \multirow{2}{*}{ Desfecho } & \multicolumn{2}{|c|}{ Preditor } & \multirow[b]{2}{*}{$\mathbf{N}$} & \multirow[b]{2}{*}{ Prev.(\%) } & \multicolumn{3}{|c|}{ Estatísticas } & \multicolumn{2}{|c|}{ IC (95\%) } \\
\hline & Est. & Comorb. & & & $x^{2}$ & $p$ & RC & Inf. & Sup. \\
\hline \multirow[t]{4}{*}{$\begin{array}{l}\text { Recuperado } \\
\text { \$ }\end{array}$} & RS & Não & 179.250 & 95,7 & $7.267,99$ & 0 & 1,149 & 1,143 & 1,155 \\
\hline & & Sim & 26.293 & 83,3 & & & & & \\
\hline & $\mathrm{SC}$ & Não & 209.364 & 96,4 & $20.917,95$ & 0 & 1,68 & 1,645 & 1,716 \\
\hline & & Sim & 3.591 & 57,4 & & & & & \\
\hline \multirow[t]{4}{*}{ Óbito } & RS & Não & 1.131 & 0,6 & $18.059,44$ & 0 & 0,046 & 0,043 & 0,049 \\
\hline & & Sim & 4.151 & 13,2 & & & & & \\
\hline & $\mathrm{SC}$ & Não & 669 & 0,3 & $60.39,77$ & 0 & 0,008 & 0,008 & 0,009 \\
\hline & & Sim & 2.271 & 36,3 & & & & & \\
\hline \multirow[t]{4}{*}{ Sintomas } & RS & Não & 103.099 & 55,1 & $6.412,35$ & 0 & 0,696 & 0,691 & 0,701 \\
\hline & & $\operatorname{Sim}$ & 24.948 & 79,1 & & & & & \\
\hline & $\mathrm{SC}$ & Não & 131.95 & 60,8 & $1.995,27$ & 0 & 0,686 & 0,679 & 0,692 \\
\hline & & Sim & 5.547 & 88,7 & & & & & \\
\hline \multirow[t]{4}{*}{ Internação } & RS & Näo & 6.813 & 3,6 & $35.900,77$ & 0 & 0,103 & 0,1 & 0,106 \\
\hline & & Sim & 11.130 & 353 & & & & & \\
\hline & $\mathrm{SC}$ & Não & 4.640 & 2,1 & $113.707,90$ & 0 & 0,023 & 0,022 & 0,023 \\
\hline & & Sim & 5.860 & 93,7 & & & & & \\
\hline
\end{tabular}

* Obs.: $\chi 2$ = Qui-Quadrado de Pearson; $\mathrm{p}<0,05 ; \mathrm{RC}=$ Razão de Chances; $\mathrm{IC}=$ Intervalo de Confiança. Fonte: Dados da pesquisa.

Observou-se que os grupos que não possuem comorbidades detém as maiores prevalências (RS: 95,7; SC: 96,4\%) para o desfecho recuperação, nos dois estados em que se dispunha de dados sobre essa variável. Para os grupos que não possuem comorbidades, a razão de chance de recuperação é de 1,149 ( $\mathrm{p}=0,000$ IC=1,143-1,155) no Rio Grande do Sul e, em Santa Catarina, é de 1,680 ( $\mathrm{p}=0,000 \mathrm{IC}=1,645-1,716)$. Ou seja, não apresentar comorbidade é uma variável preditora para 
recuperação da COVID-19, especialmente em Santa Catarina.

Pessoas que pertencem ao grupo da não-comorbidade apresentam menores taxas de prevalência e menores chances óbito: no Rio Grande do Sul a razão de chance é de 0,046 (p=0,000 IC=0,043-0,049), em Santa Catarina é de 0,008 (p=0,000 IC=0,008-0,009). Verifica-se, portanto, que comorbidade é uma variável preditora para óbito pela COVID-19, evidenciando ser um fator protetivo para pessoas que não possuem comorbidades.

As prevalências referentes a variável sintomas da COVID-19 são menores no grupo da não-comorbidade (RS: 55,1\%; SC: $60,8 \%$ ) do que no grupo da comorbidade (RS: 79,1\%; SC: 88,7\%) nos dois estados em estudo. Tal grupo apresenta menores chances de manifestarem sintomas desta doença nas seguintes taxas: no Rio Grande do Sul a razão de chance é de 0,696 ( $\mathrm{p}=0,000 \mathrm{IC}=0,691-0,701)$ e em Santa Catarina é de 0,686 ( $\mathrm{p}=0,000 \mathrm{IC}=0,679-0,692)$, denotando maior proteção para Santa Catarina. Portanto, evidencia-se que comorbidade é uma variável preditora para sintomas da COVID-19, indicando uma chance menor (fator de proteção) para o grupo da não-comorbidade nos estados pesquisados.

Nos dois estados as prevalências referentes a internação pela COVID-19 do grupo da não-comorbidade (RS: 3,6\%; SC: $2,1 \%$ ) são muito menores do que as do grupo da comorbidade (RS: 35,3\%; SC: 93,7\%). Este grupo apresenta reduzidas chances de internação nas seguintes taxas: no Rio Grande do Sul a razão de chance é de 0,103 ( $\mathrm{p}=0,000$ IC=0,100-0,106) e em Santa Catarina é de 0,023 ( $\mathrm{p}=0,000$ IC=0,022-0,023), indicando maior proteção para Santa Catarina. Assim, comorbidade se constitui em uma variável preditora para internação pela COVID-19, sinalizando uma chance menor (fator de proteção) para o grupo de não-comorbidades nos estados em questão.

\section{Discussão}

A COVID-19 (Sars-CoV-2) teve uma disseminação rápida, abrangente e expressiva em todo o mundo a partir do início de 2020. Até o final da trigésima quarta semana epidemiológica deste estudo foram confirmados 39.425 .546 casos, 1.105.403 óbitos por e uma taxa de incidência de 5.057 casos para cada 1 milhão de habitantes do planeta (Ministério da Saúde, 2020a).

Os índices de prevalência e incidência de casos ativos acumulados nos três estados da região sul, comparados em uma série temporal de 34 semanas, permitiu evidenciar taxas de prevalência e incidência similares até a décima sexta semana, quando o estado de Santa Catarina passa a mostrar uma evolução progressiva de casos novos acima dos níveis dos demais estados, até seu apogeu na vigésima segunda semana, coincidindo com a curva exponencial brasileira (Ministério da Saúde, 2020a). Na vigésima segunda semana, a tendência de queda das taxas de prevalência e incidência se acentuam nos três estados, indicando uma maior eficácia nas políticas de isolamento social.

Esta pesquisa indicou que as mulheres possuem maiores chances do que os homens de se recuperarem e de apresentarem sintomas desta doença e, consequentemente, menores chances de falecerem e serem internadas devido a COVID9. Nos três estados da região sul do Brasil tais chances se mostraram similares para a variável recuperação. No estudo de Li et al. (2020), dos 425 pacientes de COVID-19, 56\% eram do sexo masculino, indicando que homens podem ser mais suscetíveis à infecção por esta doença. Esse dado também foi observado na pesquisa de Nikpouraghdam et al. (2020), em que, dos 2.968 pacientes internados - devido a COVID-19 - existiu uma proporção de 1,93 para 1, de homens para mulheres. Petrilli et al. (2020), por sua vez, mostraram que pacientes hospitalizados são mais propensos a ser do sexo masculino do que pacientes não hospitalizados

Em termos de características biológicas, sabe-se que as mulheres, em relação aos homens, produzem respostas imunológicas mais fortes no combate a infecções, incluindo infecções virais. O sistema imunológico masculino mostra-se mais suscetível a uma série de doenças infecciosas (Ng et al., 2020). O efeito dos hormônios sexuais (estrogênio e testosterona) na resposta do sistema imunológico e engajamento, pode resultar em um sistema imunológico menos robusto nos homens e 
subsequente aumento da morbidade e mortalidade por doenças respiratórias virais (Sue, 2017).

A faixa etária de pessoas com 60 anos ou mais, neste estudo, apresentou maiores chances do que as demais faixas etárias de falecerem, de apresentarem sintomas desta doença e de serem internadas devido a esta pandemia. Esta faixa apresentou menores chances de se recuperarem da COVID-19. Quanto aos estados da região sul do Brasil tais chances desta faixa etária indicam uma menor probabilidade de recuperação para o Paraná, grau elevado de risco de óbito para Santa Catarina, alto risco para o Rio Grande do Sul na variável sintoma da COVID-19 e maior risco para Santa Catarina na variável internação por causa desta doença.

Pacientes com idade avançada acometidos com a COVID-19 têm um risco aumentado de morte (Dong et al., 2020). A idade avançada foi associada a maior gravidade e mortalidade em pacientes com COVID-19 e SARS (Jin et al., 2020; Nikpouraghdam et al., 2020). A razão de chance (odds ratio) para óbito na COVID-19 é de 3,4 para cada aumento de 10 anos na idade. Na presença de comorbidades importantes a razão de chance para óbito aumenta para 10,3 (Asfahan et al., 2020). Na população mais jovem, a obesidade é um importante sinalizador da gravidade da doença devido à deposição de gordura ectópica em vários órgãos (Deng et al., 2020).

Este estudo evidenciou que o grupo dos contaminados que não possuem comorbidades possuem maiores chances de se recuperarem desta doença; e menores chances de apresentarem sintomas de COVID-19, de falecerem e de serem internadas devido a esta pandemia do que o grupo dos que possuem comorbidades. Comparando os dois estados da região sul do Brasil, Santa Catarina oferece os melhores indicadores para este grupo, visto que ostenta a maior chance de recuperação, bem como predominância protetiva para as demais variáveis: óbito, sintomas da COVID-19 e internação.

A comorbidade está associada a um alto risco de sintomas para COVID-19. Desde o início da epidemia informações provenientes da China indicaram maiores taxas de infecção em grupos de pessoas mais velhas e com condições de comorbidades preexistentes. Pacientes com comorbidades apresentam maior probabilidade de manifestarem sintomas graves desta doença (Gao et al., 2020; Nikpouraghdam et al., 2020), associado ao maior risco de morte desses pacientes (Nikpouraghdam et al., 2020). Outros preditores de agravante da doença são as comorbidades: hipertensão, diabetes, obesidade e doenças cardiovasculares (Asfahan et al., 2020; Hernández-Garduño, 2020), bem como doenças pulmonares obstrutivas crônicas, doença renal crônica, sendo essas últimas as mais prevalentes entre pacientes hospitalizados com COVID-19 (Emami et al., 2020). Febre, tosse, fadiga e sintomas gastrointestinais foram as manifestações clínicas mais comuns, enquanto hipertensão e diabetes mellitus foram as comorbidades mais comuns (Zhang et al., 2020). Outro forte preditor de prognóstico grave em adultos jovens é a linfopenia, que afeta o sistema imunológico (Zhou et al., 2020).

\section{Considerações Finais}

Os modelos preditivos em epidemiologia permitem estratificar populações de risco no intuito de orientar a adoção de medidas públicas sanitárias e apoiar a tomada de decisões médicas diante de um contexto que exige rapidez e decisões acertadas. A descrição do comportamento de preditores para desfechos desta doença na região sul do Brasil - ao final de 34 semanas - apontam para um modelo de elucidação de alguns fatores de risco e proteção em algumas populações.

As variáveis preditoras - estado, sexo, faixa etária e comorbidade - mostrou clara associação com as variáveis desfecho - recuperação, óbitos, sintomas e internação. Desta forma, tanto o perfil epidemiológico como o cálculo dos preditores de risco e proteção para a doença realizados neste estudo sugerem que tais variáveis predizem a condição de populações contaminadas de se recuperarem, falecerem, manifestarem sintomas ou serem internadas em virtude da COVID-19.

Ao mensurar o risco epidemiológico da população feminina nesta região verificou-se que ela apresenta maior chance de recuperação do que a masculina e menor chance de falecer e ser internada devido a esta pandemia, apesar de apresentar mais sintomas do que a masculina. Em relação ao risco epidemiológico da população idosa (60 anos ou mais), constatou-se 
neste estudo, que ela ostenta maiores chances de falecer, de apresentar sintomas desta doença e de ser internada em relação aos demais, considerando a idade e apresentando poucas chances de recuperação. Quanto ao risco epidemiológico do conjunto de pessoas saudáveis que não possuem comorbidades, verificou-se nesta pesquisa, que este grupo, possui maiores chances de se recuperar da COVID-19, menor chance de apresentar sintomas de COVID-19, de ser internado ou óbito do que o grupo dos que possuem comorbidades.

Este estudo apresenta algumas limitações, entre elas, pondera-se que o banco de dados disponibilizados pelas secretarias de saúde de cada estado não possibilitou identificar a severidade da COVID-19, bem como as condições de exposição das pessoas à contaminação. $\mathrm{O}$ estudo apresenta boa validade interna, uma vez que os dados são representativos dos estratos geográficos analisados. Todavia, é preciso cautela na análise da incidência diante da possibilidade de subnotificação dos casos de COVID-19 em cada estado, devido, entre outros fatores, ao racionamento e/ou desigualdade de testes diagnósticos, a sobrecarga e variabilidade na qualidade dos serviços de saúde.

Cabe salientar que os dados relativos aos casos de COVID-19 no estado do Paraná foram limitados, não apresentando informações suficientes para a comparação de variáveis nos três estados, possibilitando apenas algumas análises. Dessa forma, não foi possível analisar as categorias sintomas, comorbidade e internação, uma vez que o banco de dados disponibilizado pela Secretaria de Estado de Saúde não apresentou esse dado.

Não se constitui como escopo deste estudo descobrir as razões pelas quais certas variáveis mostram-se como associadas ao risco ou à proteção. Nesse sentido, sugere-se que sejam realizados novos estudos que envolvam a investigação das possíveis esferas biológicas, comportamentais, estilo de vida, culturais etc., promovendo discussões multifatoriais que possibilitem tais respostas. Dessa forma, será possível o planejamento de ações mais efetivas, coerentes e resolutivas aos problemas que interferem negativamente no processo saúde-doença vivenciado pela população.

\section{Referências}

Asfahan, S., Deokar, K., Dutt, N., Niwas, R., Jain, P., \& Agarwal, M. (2020). Extrapolation of mortality in COVID-19: Exploring the role of age, sex, comorbidities and health-care related occupation. Monaldi Archives for Chest Disease, 90(2). https://doi.org/10.4081/monaldi.2020.1325

Bonita, R., Beaglehole, R., \& Kjellström, T. (2010). Epidemiologia básica. (2a ed.), Santos.

Centers for Disease Control and Prevention. (2020). Implementation of mitigation strategies for communities with local transmission COVID-19. Atlanta, GA: CDC.

Cutolo, L. R. A. (2006). Modelo Biomédico, reforma sanitária e educação pediátrica. Arquivos Catarinenses de Medicina, 35(4), $16-24$.

Deng, M., Qi, Y., Deng, L., Wang, H., Xu, Y., Li, Z., ... \& Dai, Z. (2020). Obesity as a potential predictor of disease severity in young COVID-19 patients: a retrospective study. Obesity, 28(10), 1815-1825. https://doi.org/10.1002/oby.22943

Dong, Y., Mo, X. I., Hu, Y., Qi, X., Jiang, F., Jiang, Z., \& Tong, S. (2020). Epidemiological characteristics of 2143 pediatric patients with 2019 coronavirus disease in China. Pediatrics, 145(6), e20200702. https://doi.org/10.1542/peds.2020-0702

Emami, A., Javanmardi, F., Pirbonyeh, N., \& Akbari, A. (2020). Prevalence of underlying diseases in hospitalized patients with COVID-19: a systematic review and meta-analysis. Archives of academic emergency medicine, 8(1).

Gao, Q., Hu, Y., Dai, Z., Xiao, F., Wang, J., \& Wu, J. (2020). The epidemiological characteristics of 2019 novel coronavirus diseases (COVID-19) in Jingmen, Hubei, China. Medicine, 99(23), e20605. https://doi.org/10.1101/2020.03.07.20031393

Giordano, G., Blanchini, F., Bruno, R., Colaneri, P., Di Filippo, A., Di Matteo, A., \& Colaneri, M. (2020). Modelling the COVID-19 epidemic and implementation of population-wide interventions in Italy. Nature medicine, 26(6), 855-860. https://doi.org/10.1038/s41591-020-0883-7

Governo do Estado do Paraná. (2020). Boletim - Informe Epidemiológico Coronavírus (COVID-19). Secretaria da Saúde do Estado do Paraná.

Governo do Estado do Rio Grande do Sul. (2020). Painel Coronavírus RS. Secretaria da Saúde do Estado do Rio Grande do Sul.

Governo do Estado de Santa Catarina. (2020). COVID-19 - Casos Confirmados. Base de dados do Governo do Estado - BOAVISTA, Centro de Operações de Emergência em Saúde (COES). Portal de Dados Abertos do Estado de Santa Catarina.

Guimarães, R. B., Catão, R. D. C., Martinuci, O. D. S., Pugliesi, E. A., \& Matsumoto, P. S. S. (2020). O raciocínio geográfico e as chaves de leitura da Covid19 no território brasileiro. Estudos Avançados, 34(99), 119-140. https://doi.org/10.1590/s0103-4014.2020.3499.008 
Hernández-Garduño, E. (2020). Obesity is the comorbidity more strongly associated for Covid-19 in Mexico. A case-control study. Obesity research \& clinical practice, 14(4), 375-379. https://doi.org/10.1016/j.orcp.2020.06.001

Instituto Brasileiro de Geografia e Estatística (IBGE) (2020). Estimativas da população residente no Brasil e Unidades da Federação com data de referência em $1^{\circ}$ de julho de 2020 .

Jin, J. M., Bai, P., He, W., Wu, F., Liu, X. F., Han, D. M., \& Yang, J. K. (2020). Gender differences in patients with COVID-19: focus on severity and mortality. Frontiers in public health, 8,152 . https://doi.org/10.3389/fpubh.2020.00152LI

Li, Q., Guan, X., Wu, P., Wang, X., Zhou, L., Tong, Y., \& Feng, Z. (2020). Early transmission dynamics in Wuhan, China, of novel coronavirus-infected pneumonia. New England journal of medicine. https://doi.org/10.1101/2020.02.23.20026864.

Ministério da Saúde. (2020a). Boletim Epidemiológico n. 36. Doença pelo Coronavírus 2019 (COVID-19).

Ministério da Saúde. (2020b). Boletim Epidemiológico Especial da Semana Epidemiológica 29 (12 a 18/07/20). Secretaria de Vigilância em Saúde do Ministério da Saúde. Brasília - DF.

Ng, J., Bakrania, K., Russell, R., \& Falkous, C. (2020). Covid-19 mortality rates by age and gender: why is the disease killing more men than women. RGA.

Nikpouraghdam, M., Farahani, A. J., Alishiri, G., Heydari, S., Ebrahimnia, M., Samadinia, H., \& Bagheri, M. (2020). Epidemiological characteristics of coronavirus disease 2019 (COVID-19) patients in IRAN: A single center study. Journal of Clinical Virology, 127, 104378. https://doi.org/10.1016/j.jcv.2020.104378.

Pereira, M. G. (2013). Epidemiologia: teoria e prática. Guanabara Koogan.

Pereira, A. S., Shitsuka, D. M., Parreira, F. J., \& Shitsuka, R. (2018). Metodologia da Pesquisa Científica. UFSM.

Petrilli, C. M., Jones, S. A., Yang, J., Rajagopalan, H., O'Donnell, L. F., Chernyak, Y., \& Horwitz, L. I. (2020). Factors as sociated with hospitalization and critical illness among 4,103 patients with COVID-19 disease in New York City. MedRxiv. https://doi.org/10.1136/bmj.m1966

Singh, A. K., Gillies, C. L., Singh, R., Singh, A., Chudasama, Y., Coles, B., \& Khunti, K. (2020). Prevalence of co-morbidities and their association with mortality in patients with COVID-19: a systematic review and meta-analysis. Diabetes, Obesity and Metabolism, 22(10), 1915-1924. https://doi.org/10.1111/dom.14124

Sue, K. (2017). The science behind “man flu”. bmj, 359. https://doi.org/10.1136/bmj.j5560

Verity, R., Okell, L. C., Dorigatti, I., Winskill, P., Whittaker, C., Imai, N., \& Ferguson, N. M. (2020). Estimates of the severity of coronavirus disease 2019: a model-based analysis. The Lancet infectious diseases, 20(6), 669-677. https://doi.org/10.1016/S1473-3099(20)30243-7

World Health Organization (WHO). (2020). Coronavirus disease (COVID-19) outbreak. Geneva/WHO.

Zhang, J. J., Dong, X., Cao, Y. Y., Yuan, Y. D., Yang, Y. B., Yan, Y. Q., \& Gao, Y. D. (2020). Clinical characteristics of 140 patients infected with SARS-CoV-2 in Wuhan, China. Allergy, 75(7), 1730-1741. https://doi.org/10.1111/all.14238

Zheng, Z., Peng, F., Xu, B., Zhao, J., Liu, H., Peng, J., \& Tang, W. (2020). Risk factors of critical \& mortal COVID-19 cases: A systematic literature review and meta-analysis. Journal of Infection. https://doi.org/10.1016/j.jinf.2020.04.021

Zhou, C., Huang, Z., Tan, W., Li, X., Yin, W., Xiao, Y., \& Hu, Y. (2020). Predictive factors of severe coronavirus disease 2019 in previously healthy young adults: a single-center, retrospective study. Respiratory research, 21(1), 1-8. https://doi.org/10.1186/s12931-020-01412-1 